\title{
Mental Illness and Opioid Epidemic in the United States
}

\author{
Evelio Velis, $M D, P h D$ \\ Selene Borras, BBA, MIB \\ Sumera Khaja, BS \\ Barry University, College of Nursing and Health Sciences, \\ Miami Shores, FL, U.S.A.
}

Doi: 10.19044/esj.2018.c4p11～URL:http://dx.doi.org/10.19044/esj.2018.c4p11

\begin{abstract}
Introduction: According to the World Health Organization (WHO), mental health is defined as a state of well-being in which every individual realizes his or her own potential, can cope with the normal stresses of life, can work productively and fruitfully, and is able to make a contribution to her or his community. A drug overdose is defined, by the Medical Dictionary, as the accidental or intentional use of a drug or medicine in an amount that is higher than is normally used. Accidental drug overdose may be the result of misuse of prescription medicines or commonly used medications like pain relievers and cold remedies. Overdose with opiate drugs causes sedation, low blood pressure, slowed heart rate, and slowed breathing. A serious risk is that the patient will stop breathing. Methods: A multiple correlational analysis was conducted to explore the potential strength, direction and significant of relationship between selected indicators related to mental illness, socioeconomic factors and drug related deaths in the United States, at the state level. A level of significance of 0.05 was selected for all tests of significance. Conclusion: There is a strong and significant correlation at the state level between the mental illness, major depressive disorders, drug use/abuse and drug overdose related deaths. Veterans living in the state and un-insured individuals are significantly correlated with drug overdose related deaths. No significant correlation was identified between socio-economic factors typically associated with most health issues such as poverty and the current opioid epidemic affecting our nation.
\end{abstract}

Keywords: Mental illness, depression, post-traumatic stress disorder, drug overdose and abuse, drug related deaths, socio-economic factors 


\section{Introduction}

\section{Mental Health}

According to the World Health Organization (WHO), mental health is defined as a state of well-being in which every individual realizes his or her own potential, can cope with the normal stresses of life, can work productively and fruitfully, and is able to make a contribution to her or his community (WHO, 2014). ${ }^{1}$

In their article "Toward a new definition of mental health" published in World Psychiatry, the authors propose a new definition: "Mental health is a dynamic state of internal equilibrium which enables individuals to use their abilities in harmony with universal values of society. (Galderisi et al., 2015). ${ }^{2}$

The Mayo Clinic referred to mental illness as to a "wide range of mental health conditions - disorders that affect your mood, thinking and behavior" (Mayo Clinic, 2015) .

The National Center for Health Statistics, in its National Health Interview Survey Early Release Program, reported that in 2016, 3.6\% (95\% confidence interval $=3.35 \%-3.88 \%$ ) of adults aged 18 and over had experienced serious psychological distress during the past 30 days. The percentage of adults who had experienced serious psychological distress during the past 30 days increased, from 2014 through 2016, the percentage of adults who had experienced serious psychological distress during the past 30 days increased from $3.1 \%$ to $3.6 \%$ (Reinberg, 2017) .

\section{Depression}

According to the American Psychiatry Association (APA), depression (major depressive disorder) is a common and serious medical illness that negatively affects how you feel, the way you think and how you act. Depression causes feelings of sadness and/or a loss of interest in activities once enjoyed. It can lead to a variety of emotional and physical problems and can decrease a person's ability to function at work and at home (APA, 2017). ${ }^{5}$

\section{Post-traumatic stress disorder (PTSD)}

Post-traumatic stress disorder is defined by The Mayo Clinic as a mental health condition that's triggered by a terrifying event - either experiencing it or witnessing it. Symptoms may include flashbacks, nightmares and severe anxiety, as well as uncontrollable thoughts about the event. According to The Mayo Clinic, most people who go through traumatic events may have temporary difficulty adjusting and coping, but with time and good self-care, they usually get better. If the symptoms get worse, last for months or even years, and interfere with your day-to-day functioning, you may have PTSD. 
Getting effective treatment after PTSD symptoms develop can be critical to reduce symptoms and improve function. Some of the traumatic events leading to the development of PTSD include: combat exposure, childhood physical abuse, sexual violence, physical assault, an accident, among others.

PTSD is highly prevalent among military personnel, especially after they have participated in military/war conflicts. The prevalence of PTSD in deployed U.S. military personnel may be as high as $14-16 \%$, twice higher than the estimate for the general population (7\%-8\%) (Gates et.al, 2012). ${ }^{6}$

PTSD may also increase the risk of other mental health problems such as: depression and anxiety, issues with drugs and alcohol use and suicidal thoughts and actions (APA, 2013). ${ }^{7}$

\section{Drug Overdose}

Mental illness has been considered an important risk factor for substance abuse and drug overdose deaths. In an article published in The Journal of Clinical Psychiatry, the authors concluded that mental illness may have contributed to substance abuse associated with deaths and recommend that clinicians should screen for mental illness when prescribing opioids and recommend psychotherapy as an adjunct or an alternate to pharmacotherapy (Toblin et.al, 2010). ${ }^{8}$

A drug overdose is defined by the Medical Dictionary as the accidental or intentional use of a drug or medicine in an amount that is higher than is normally used. Accidental drug overdose may be the result of misuse of prescription medicines or commonly used medications like pain relievers and cold remedies. Overdose with opiate drugs causes sedation (sleepiness), low blood pressure, slowed heart rate, and slowed breathing. Pinpoint pupils, where the black centers of the eyes become smaller than normal, are common in opiate overdose. However, if other drugs are taken at the same time as the opiates, they may counteract this effect on the pupils. A serious risk is that the patient will stop breathing (GEM, 2008). ${ }^{9}$

According to the Centers for Disease Control and Prevention (CDC), there has been a significant increase in overdose deaths involving opioids, especially during the last five years. See Figure below from CDC/NCHS, National Vital Statistics System, 2017 (CDC, 2017). ${ }^{10}$

In March 2018, at the Opioid Epidemic: The Crisis Next Door event, President Donald J. Trump, in October 2017, directed the Department of Health and Human Services to declare the opioid crisis a public health emergency, taking action to address a rapidly escalating epidemic of drug use. The President, said that "no part of our society -not young or old, rich or poor, urban or rural- has been spared this plague of drug addiction and this horrible, horrible situation that's taken place with opioids" (Crisis Next Door, 2017). ${ }^{11}$ 
Data recently published by the White House claims that drug overdoses are now the leading cause of injury death in the United States, outnumbering traffic crashes or gun-related deaths. According to preliminary analysis, more than 64,000 lives were lost to drug overdoses in 2016, devastating American families and communities, represents a rate of 175 deaths a day in 2016. The White House reports that since 2000, over 300,000 Americans have died from overdoses involving opioids (Crisis Next Door, 2017)..$^{11}$

\section{Objectives/Purpose}

The purpose of this cross-sectional study is to explore the incidence and prevalence of mental health conditions, such as PTSD and major depression disorders and their association with drug addiction and its consecuenses in the United States at the state level. The study also aims to identify states with the highest drug overdose related mortality and concuomitant factors. Knowing that this is a very complex public health issue with no simple or easy solution identifying factors associated with mental illnes and drug overuse and abuse could facilitate the development of effective public health policies fundamentally at the primary prevention level.

\section{Learning Outcomes}

Identify the strength, direction and significance of the relationship between incidence and prevalence of mental health conditions such as depression, drug addiction/abuse, drug related mortality and socio-economic factors in the United States, at the state level.

\section{Methods:}

\section{Sources of data}

Indicators related to incidence and prevalence of mental illness, drug overuse/abuse and related mortality were selected and collected from the Centers for Disease Control and Prevention (CDC) while socio-economic related variables/indicators were extracted from the Census Bureau; we used the analyses data reported from the year 2016. Other sources of information were used and properly identified throughout the text. The study's selected variables associated with mental illness are: prevalence of serious mental illness including PTSD and major depressive disorder. The number of deaths related to drug overdose, pain killers misuse and heroin use during past year are the selected variables associated with drug overuse and abuse. Other socioeconomic related variables such as poverty, educational level, veterans living in the state and veterans with potential or provisional PTSD were also collected and analyzed. 


\section{Statistical Analysis}

A multiple correlational analysis was conducted to explore the potential strength, direction and significant of relationship between the incidence and prevalence of depression, other mental health conditions, drug overuse and abuse and related deaths in the United States at the state level. An evaluation of selected socio-economic indicators such as poverty and educational level and their relationship with mental illness and drug addiction was also performed. The Statistical Package for Social Sciences (SPSS 22®) was used to analyze collected data. A level of significance of 0.05 was selected for all tests of significance.

\section{Results}

The northeastern states of West Virginia, New Hampshire, Maryland, Ohio, Massachusetts and District of Columbia shared the highest number of mental illness, depression and opioid overdose death rate in the nation. Table 1 .

Table 1. Top six states reporting Serious Mental Illness, Major Depressive Episode and Opioid Overdose Death Rate

\begin{tabular}{cccc}
\hline State & $\begin{array}{c}\text { Serious Mental } \\
\text { Illness }\end{array}$ & $\begin{array}{c}\text { Major Depressive } \\
\text { Episode }\end{array}$ & $\begin{array}{c}\text { Opioid Overdose } \\
\text { Death Rate }\end{array}$ \\
\hline West Virginia & 1068 & 1786 & 43 \\
New Hampshire & 650 & 1132 & 36 \\
Maryland & 626 & 1026 & 30 \\
Ohio & 570 & 979 & 33 \\
Massachusetts & 453 & 693 & 30 \\
District of Columbia & 416 & 677 & 30 \\
\hline
\end{tabular}

Significant increases in drug overdose death rates from 2015 to 2016 were seen in the Northeast, Midwest and South Census Regions, according to CDC reports. The top ten States with the highest and statistically significant increases in drug overdose death rates included District of Columbia, Maryland, Florida, Pennsylvania, New Jersey, Delaware, Maine, Virginia, Illinois and Vermont. Table 2. 
Table 2. Top ten states reporting the highest and significant increase in drug overdose death rate

\begin{tabular}{ccc}
\hline No & State & $\begin{array}{c}\text { Percent Change } \\
\text { from 2015 to 2016 }\end{array}$ \\
\hline 1 & District of Columbia & 108.6 \\
2 & Maryland & 58.9 \\
3 & Florida & 46.3 \\
4 & Pennsylvania, & 44.1 \\
5 & New Jersey & 42.3 \\
6 & Delaware & 40.0 \\
7 & Maine & 35.4 \\
8 & Virginia & 34.7 \\
9 & Illinois & 34.0 \\
10 & Vermont & 32.9 \\
\hline
\end{tabular}

\section{Correlation Analysis}

A strong, direct and significant correlation was identified between serious mental illness, major depressive disorder and the number of deaths related to drug overdose in all states of the union. An even stronger correlation was confirmed between mental illness, serious mental illness, major depressive disorder and the number of deaths related to drug overdose when the top 20 states with highest opioid death rate were selected. Table 3.

Table 3. Pearson Correlation Coefficients and significant level from correlation analyses.

Drug Overdose Number of Deaths

\begin{tabular}{ccc}
\cline { 2 - 3 } & All States & Top 20 States $\dagger$ \\
\hline Serious Mental Illness & $.887^{* *}$ & $.953^{* *}$ \\
Major Depressive Episode & $.882^{* *}$ & $.966^{* *}$ \\
\hline
\end{tabular}

$*$ Note $* \mathrm{p}<.05, * * \mathrm{p}<.01, * * * \mathrm{p}<.001$

$\dagger$ Top 20 States with highest opioid death rate in 2016

There is a strong, direct and highly significant correlation between the prevalence of serious mental illness and the number of deaths due to drug overdose, $r(50)=.89, p<0.01$. Major depressive disorder is directly and significantly correlated with the number of deaths related to drug overdose, $r(50)=.97, p<.01$. Figure 1 and 2 . 
Figure 1. Scatter diagrams showing the correlation between cases of serious mental illness reported and drug overdose number of deaths reported per state.

US, 2016.

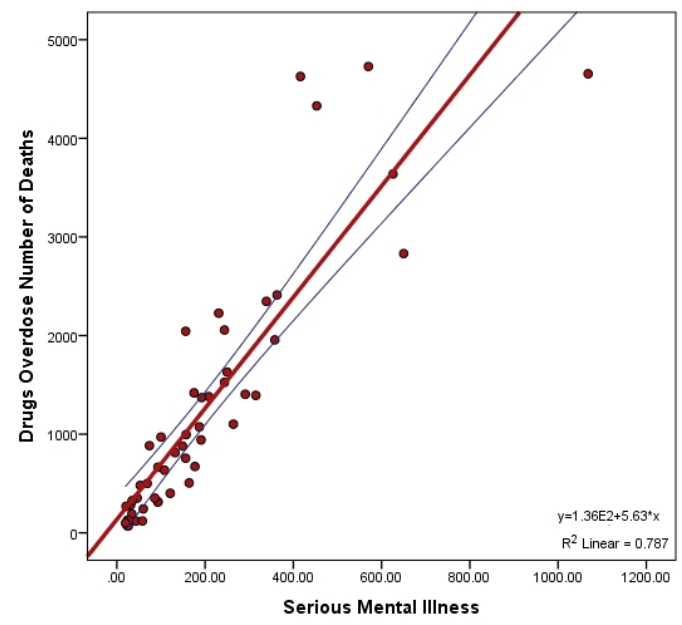

Figure 2. Scatter diagrams showing the correlation between major depressive disorder and drug overdose number of deaths reported per state. US, 2016.

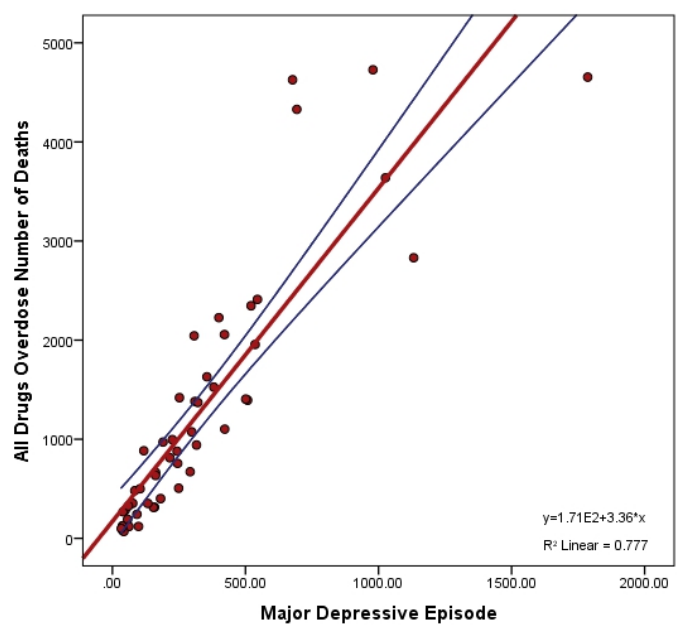

The number of pain killer misuse reported in 2016 is significantly and directly related to the number of drug overdose related deaths reported during the same year, $r(50)=.83, p<.01$. The correlation between the misuse of heroin during the past year (2016) and the number of drug overdose related deaths was even stronger, $r(50)=.90, p<.01$. Figure 3 and 4 . 
Figure 3. Scatter diagrams showing the correlation between pain killers misused during past year and drug overdose number of deaths reported per state.

US, 2016.

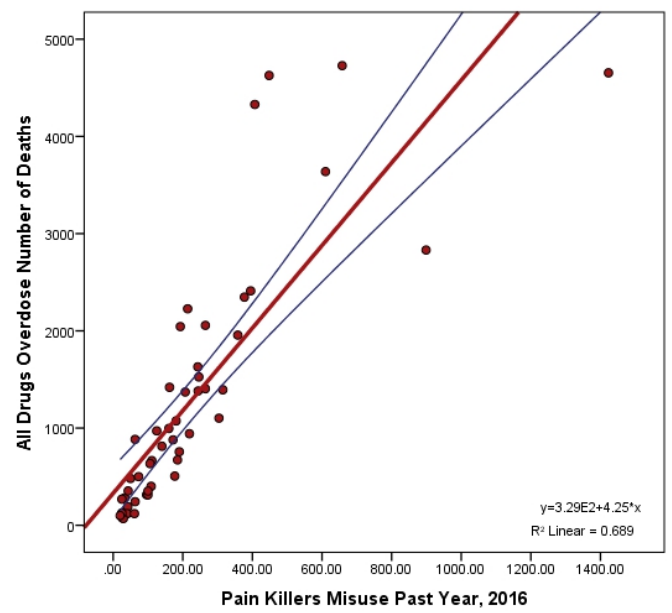

Figure 4. Scatter diagrams showing the correlation between Heroin misuse during past year and drug overdose number of deaths reported per state. US,

2016.

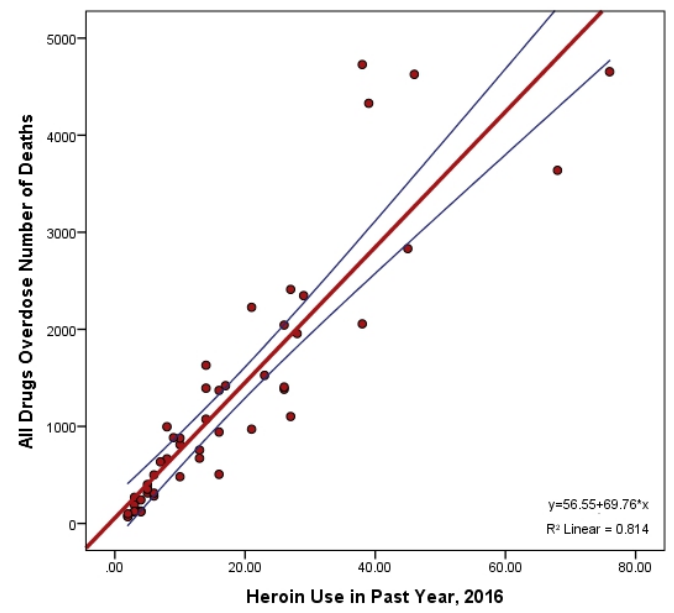

An interesting finding encountered during our exploratory investigation was the strong, direct and highly significant correlation between 
the number of deaths reported due to drug overdose and the veteran population living in the state, $r(50)=.86, p<.01$. Table 4 and Figure 5.

When selecting the top 20 states with the highest opioid overdose related death, the correlation between the number of deaths reported due to drug overdose and the veteran population living in the state is even stronger. See Figure 6.

Figure 5. Scatter diagrams showing the correlation between the population of veterans living in the state and drug overdose number of deaths reported per state.

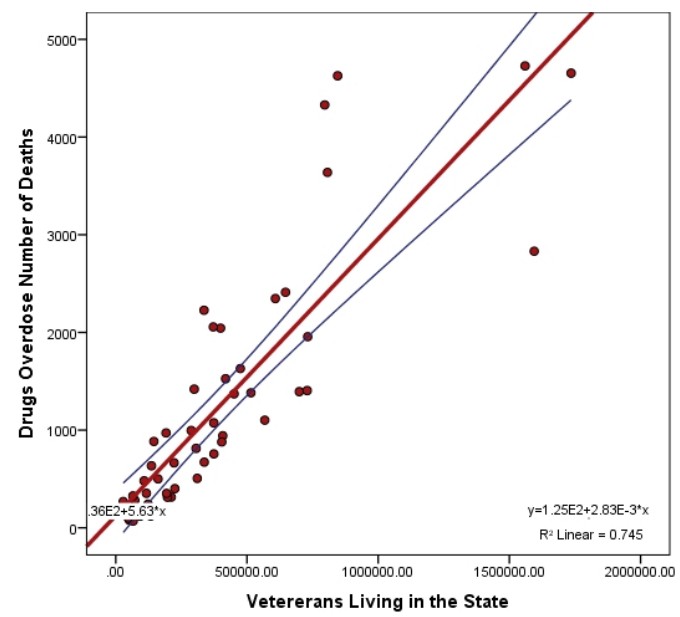

Figure 6. Scatter diagrams showing the correlation between population of veterans living in the state and drug overdose number of deaths reported per state.*

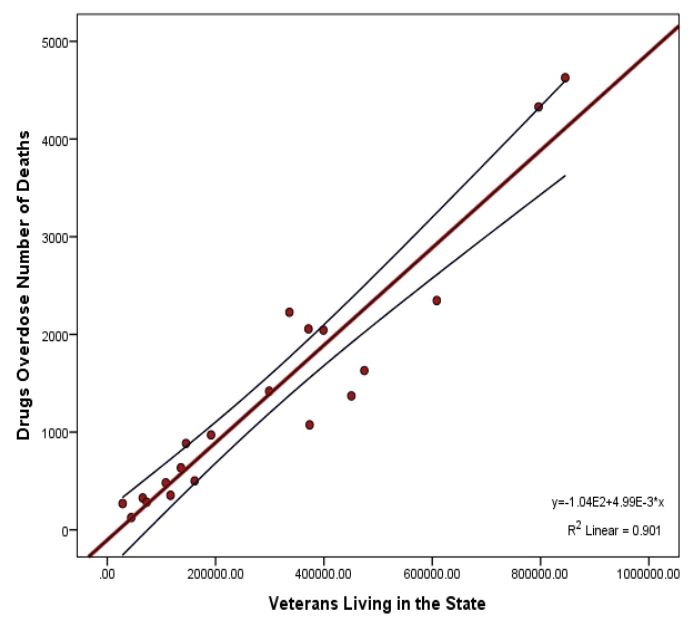

* Top 20 states with highest opioid overdose death rates are selected 
The prevalence of veterans with potential or provisional PTSD is directly and significant correlated with the number of deaths reported due to drug overdose, $r(50)=.71, p<.01$. Table 4.

Table 4. Pearson Correlation Coefficients and significant level from correlation analyses.

\begin{tabular}{cc}
\hline Indicator & $\begin{array}{c}\text { Drug Overdose Number of } \\
\text { Deaths } 2016\end{array}$ \\
\hline Veteran Population Living in the State & $.863^{* *}$ \\
Veterans with Potential or Provisional PTSD (Prevalence) & $.714^{* *}$ \\
\hline$*$ Note $* \mathrm{p}<.05, * * \mathrm{p}<.01, * * * \mathrm{p}<.001$ &
\end{tabular}

Socio-economic factors such as poverty rate $[r(50)=.12, p=.39]$ and median income per family $[r(50)=.02, p=.90]$, typically associated with most health issues in US, do not appear to be significantly related to the current drug/opioid epidemic affecting our nation. Educational level continues to be a factor related to drug misuse and abuse. There was identified a significant but inverse relationship between heroin use during the past year (2016) and the proportion of the adult population who had completed high school or a higher educational level, $r(50)=-.35, p<.05$.

\section{Discussion}

According to the Centers for Disease Control and Prevention (CDC), opioid deaths have increased five-fold from 1999 to 2016 due to the use of prescription and/or illicit opioid drugs, that have contributed to over 42,000 deaths in 2016 (CDC, 2017) ${ }^{12}$. Data from the CDC further indicates that the highest rates from deaths due to drug overdose were in West Virginia (52.0 per 100, 000), Ohio (39.1 per 100, 000), New Hampshire (39.0 per 100,000), Pennsylvania (37.9 per 100, 000) and Kentucky $(33.5$ per 100,000) (CDC, $2017)^{12}$. These states tend to have about a $25 \%$ of their population in manual labor, which could lead that area to have more need for opioids to deal with on the job related injuries (Industries in the Northeast, 2015). ${ }^{13}$

Specifically in West Virginia, about eight percent of the state occupation is in construction which is 54\% higher than the rest of the United States (Occupations in West Virginia, 2015) ${ }^{14}$. Our findings are concurrent with these reports; the north eastern states of West Virginia, New Hampshire, Maryland, Ohio, Massachusetts and District of Columbia shared the highest number of mental illness, depression and opioid overdose death rate in the nation.

We identified a strong, direct and highly significant correlation between the prevalence of serious mental illness, major depressive disorders and the number of deaths due to drug overdose. According to national surveys, 75 percent or more of patients with mental illness struggle with drug and/or alcohol problems. ${ }^{1}$ 
Our study found that the number of pain killer misuse reported in 2016 is significantly and directly related to the number of drug overdose related deaths reported during the same year. Dr. Anna Lembke, in her book "Drug Dealer, MD: How doctors were duped, patients got hooked and why it's so hard to stop", argued the thesis that prescription drugs has turned the gateway to addition especially in young individuals. ${ }^{15}$ On November 1, 2011, the Centers for Disease Control and Prevention (CDC), the agency of the government responsible for protecting Americans from major health threats, declared a "prescription drug epidemic"; and the CDC was unequivocal about what had caused this epidemic: "prescription opioid painkillers and psychotherapeutic drugs being prescribed more widely by physicians."2

Evidence suggests that drug overdoses related to opioids are underreported by as much as 24 percent, which would raise the estimated 2015 opioid overdose death toll to over 40,000. Since 2000, over 300,000 Americans have died from overdoses involving opioids. (The Crisis Next Door, 2017). ${ }^{11}$

In our study, we found that the direct and significant correlation between the misuse of heroin during the past year (2016) and the number of drug overdose related deaths was even stronger. According to The Opioid Epidemic and the Labor Market report, the decrease in the price of heroin also corresponds to a 143 percent increase in heroin seizures between 2010 and 2015 (FRBCL, 2017). ${ }^{16}$

An interesting finding encountered during our exploratory investigation was the strong, direct and highly significant correlation between the number of deaths reported due to drug overdose, the veteran population living in the state and the prevalence of veterans with potential or provisional PTSD. The Combat Posttraumatic Stress Disorder, Substance Use Disorders, and Traumatic Brain Injury reported that PTSD may also increase the risk of developing a substance use disorders (SUD) because individuals may use substances in an attempt to relieve symptoms of PTSD (Brady et.al, 2009). ${ }^{17}$

The access and quality of health services offered and provided to our veterans have been a focus of attention during the past decades. Recently, the Veterans Affairs Department Secretary, David Shulkin, was fired and new administration will be in place soon. Based on our results, we believe that our veterans are in the center of the opioid epidemic, suffering from the dire consequences of the combination of diverse mental illness and drug addiction. More resources should be placed in the Veterans Health Administration, better access to health services should be guaranteed to our veterans and advanced and more efficient management should be put into action.

Socio-economic factors, typically associated with most health issues in US such as poverty rate and median income per family, do not appeared to be particularly related to the current drug/opioid epidemic affecting our nation. 
The Opioid Epidemic and the Labor Market report conclude that we need better data to understand the opioid epidemic, and that the relationship between drug use and the labor market warrants further study. (FRBCL, 2017). ${ }^{16}$

We believe that more specific data should be available at the local level, the access to current and valid data would be highly benefitial to the local health departments and health officials in order to have a better picture of the mental illnes and opioid overuse in their communities; thus the effecticveness of interventions will significantly increase.

\section{Conclusion}

A strong and significant correlation between the incidence of mental illness, major depressive disorders, drug misuse/abuse and drug overdose related deaths was identified at the nation's state level.

No significant correlation was identified between socio-economic factors such as poverty rate and median income per family, typically associated with most health issues, and the current drug/opioid epidemic affecting our nation. However, the educational level appeared to be a protective factor related to heroin use and abuse.

Mental illness and its strong association with drug overuse and abuse is a complex and multifactorial public health issue, we understand that correlation does not necessarily imply cause and effect relationship, but we recommend, based on results from our study, that the factors selected and analyzed in this study should be taking into consideration by clinicians, government and public health officials when developing strategies to effectively reduce incidence, prevalence and mortality associated with drug/opioid epidemic in US. These factors must be taken into carefully consideration when developing and implementing interventions to control this public health crisis. It is a monumental task; we should be prepared for a long standing fight.

\section{References:}

1. World Health Organization. (2014, August). Mental health: a state of well-being.

Retrieved

from

http://www.who.int/features/factfiles/mental_health/en/

2. Galderisi, S., Heinz, A., Kastrup, M., Beezhold, J., \& Sartorius, N. (2015). Toward a new definition of mental health. World Psychiatry, 14(2), 231-233.

3. Mayo Clinic. (2015, October 13). Mental illness. Retrieved from https://www.mayoclinic.org/diseases-conditions/mentalillness/symptoms-causes/syc-20374968 
4. Reinberg, Steven. (2017, March 29). Heroin epidemic expand its grip on America. HealthDay News. Retrieved from www. WebMD.com.

5. American Psychiatric Association. (2017, January). What is depression? Retrieved from https://www.psychiatry.org/patientsfamilies/depression/what-is-depression

6. Gates, M. A., Holowka, D. W., Vasterling, J. J., Keane, T. M., \& Marx B. P. (2012). Posttraumatic Stress Disorder in Veterans and Military Personnel: Epidemiology, Screening, and Case Recognition. Psychological Services, Vol. 9, No. 4, 361-382.

7. Posttraumatic stress disorder. In: Diagnostic and Statistical Manual of Mental Disorders DSM-5. 5th ed. Arlington, Va.: American Psychiatric Association; 2013. http://www.psychiatryonline.org. Accessed Dec.13, 2016.

8. Toblin, R. L., Paulozzi, L. J., Logan, J. E., Hall, A. J., \& Kaplan, J. A. (2010). Mental Illness and Psychotropic Drug Use Among Prescription Drug Overdose Deaths. The Journal of Clinical Psychiatry, 71(04), 491-496. doi:10.4088/jcp.09m05567blu

9. Gale Encyclopedia of Medicine (drug overdose). (n.d.). (2008). Retrieved March $28 \quad 2018$ from https://medicaldictionary.thefreedictionary.com/drug+overdose.

10. US Department of Health and Human Services (2017). CDC/NCHS, National Vital Statistics System, Mortality.

11. The Crisis Next Door: President Donald J. Trump is Confronting an Opioid Crisis More Severe Than Original Expectations (2017). Retrieved from https://www.whitehouse.gov/briefingsstatements/crisis-next-door-president-donald-j-trump-confrontingopioid-crisis-severe-original-expectations/

12. Centers for Disease Control (2017). Understanding the epidemic. Retrieved from https://www.cdc.gov/drugoverdose/epidemic/index.html

13. Industries in the Northeast. (2015, April). Retrieved from https://statisticalatlas.com/region/Northeast/Industries

14. Occupations in West Virginia (2015, April). Retrieved from https://statisticalatlas.com/state/West-Virginia/Occupations

15. Lembke, A. (2016). Drug Dealer, MD: How Doctors were duped, patients got hooked and why it's so hard to stop. Baltimore. MD. Johns Hopkins University Press.

16. Federal Reserve Bank of Cleveland. (2017, September). The opioid epidemic and labor market. Retrieved from https://www.clevelandfed.org/newsroom-andevents/publications/economic-commentary/2017-economiccommentaries/ec-201715-opioids.aspx 
17. Brady, K. T., Tuerk, P., Back, S. E., Saladin, M. E., Waldrop, A. E., \& Myrick, H. (2009). Combat Posttraumatic Stress Disorder, Substance Use Disorders, and Traumatic Brain Injury. Journal of Addiction Medicine, $3(4)$, 179-188. http://doi.org/10.1097/ADM.0b013e3181aa244f 